\title{
Design of an On-Chip Linear-Assisted DC-DC Voltage Regulator
}

\author{
Jordi Cosp-Vilella, and Herminio Martínez-García \\ College of Industrial Engineering of Barcelona (EUETIB) \\ Department of Electronics Engineering \\ Technical University of Catalonia (UPC). BarcelonaTech \\ C/ Comte d'Urgell, $n^{\circ} 187$ \\ 08036 - Barcelona. SPAIN \\ \{jordi.cosp, herminio.martinez\}@upc.edu
}

\begin{abstract}
This article shows the design of an on-chip CMOS linear-assisted DC-DC regulator. It results a good alternative topology to classic switching DC-DC power converters. In the presented technique, an auxiliary linear regulator is used to cancel the output voltage ripple and provides fast responses for load and line variations. On the other hand, a switching converter, connected in parallel, allows supplying almost the whole output current demanded by the load. The objective of this linear-assisted regulator or hybrid topology is to achieve a high efficiency of switching converters, with suitable load and line regulation features, typical of linear regulators. In this kind of on-chip applications, CMOS is the current prevailing technology. Thus, in order to implement on-chip power supply systems and on-chip power management systems with low-tomedium current consumption, this structure has good features.
\end{abstract}

\section{INTRODUCTION}

Series linear regulators have been structures widely used for decades in power supply systems providing supplies with low or moderate currents and consumes [1], [2]. These voltage regulators have several advantages that lead their use. However, they suffer from some serious disadvantages: the efficiency of these structures hardly exceeds 50\%. Their series-pass transistor has to support all current required by the load and, therefore, in high power applications, this component has to be dimensioned (both electrically and thermically) in order to dissipate high power. Since the minimum permissible dropout voltage of a linear regulator defines the maximum achievable efficiency, in order to improve the efficiency of this circuit, the use of LDO (low dropout) regulators has been extended in last years. Although this increase in efficiency is achieved, the cost of a compromise in stability of the regulator is paid. However, nowadays, the emphasis on efficiency has made LDO regulators the most popular class of linear regulators [3], [4].

The alternative to linear regulators is DC-DC switching converters. Many of these structures [5], [6] have been designed, and their main advantage is their high efficiency that, although not reaching $100 \%$ due to omnipresent circuit losses, is near this optimal figure. However, they present some important problems: the design and implementation of this sort of converters is a more complex process than in linear regulators, especially their control loops. In addition, the intrinsic switched nature of these converters increments EMIs in neighboring electronic systems due to significant ripples in currents and voltages in the converter. In order to minimize the aforementioned output ripple voltage, it is necessary to include both a bulky and expensive inductor and an output capacitor.

Linear-switching hybrid regulators (also known as linearswitching hybrid regulators or converters) are compact circuit topologies that preserve the well-known advantages of the two typical alternatives for the implementation of DC/DC voltage regulators, namely, achieving both moderately high efficiencies - by virtue of the switching regulator- together with fast wideband ripple-free regulation -thanks to the linear regulator-. They are circuit topologies of strong interest when designing power supplies requiring as design specifications both: (1) High slew-rate of the output current, and, (2) high current consumption by the output load. This is the case of systems based on modern microprocessors and DSPs, where both requirements converge [7], [8]. This interest is also applicable to wideband adaptive supply of RF power amplifiers.

In this paper, a linear-assisted strategy is applied to an onchip regulator. CMOS technology has rapidly embraced the field of analog integrated circuits, providing low-cost, high performance solutions and tending to dominate the market of integrated circuits. Thus, in this article, a CMOS design of a DC-DC regulator based on a linear-assisted topology is presented for an on-chip application. The design must guarantee the electric power supply for a critical load that needs a constant $1.1 \mathrm{~V}$ supply. This load is an analog design (included in the same chip) that consists of a continuous time filter for MEMS signal filtering with its central-frequency and quality-factor control loops. The regulator input voltage (chip input voltage) can vary from $1.6 \mathrm{~V}$ to $1.8 \mathrm{~V}$. 
With the compact structure designed, the output ripple is negligible without any output capacitor for a wide range of output currents. In addition, some of the aforementioned disadvantages are minimized as the low efficiency and the high power dissipation present in linear regulators, or the complexity in the design of the control for switching converters.

\section{Proposed ARQUiteCtURE FOR THE ON-ChIP LINEAR- ASSISTED DC-DC REGULATOR}

Let us consider a series linear regulator that supplies a load $R_{L}$ with constant output voltage $V_{\text {out }}$. With the objective of reducing the dissipated power in the series-pass transistor of the regulator, it is also necessary to reduce the current through the regulator as far as possible below a maximum value. In case the load current should be higher than this maximum value, it is possible to introduce a buck (or step-down) switching converter into the structure. This second block will be connected in parallel with the first one, and will provide the excess current that the series linear regulator fails to supply. The original idea, which is presented and analyzed in [9], [10] and [11], needs a clock signal for the switching converter.

Nevertheless, the proposed configuration in this paper (Fig. 1) makes use of the analog hysteretic comparator $C M P_{1}$ that controls the conduction or cut of transistor $Q_{1}$, and fixes its switching frequency. Notice that the main objective of the switching converter is to provide the excess of current that the linear regulator fails to supply. Consequently:

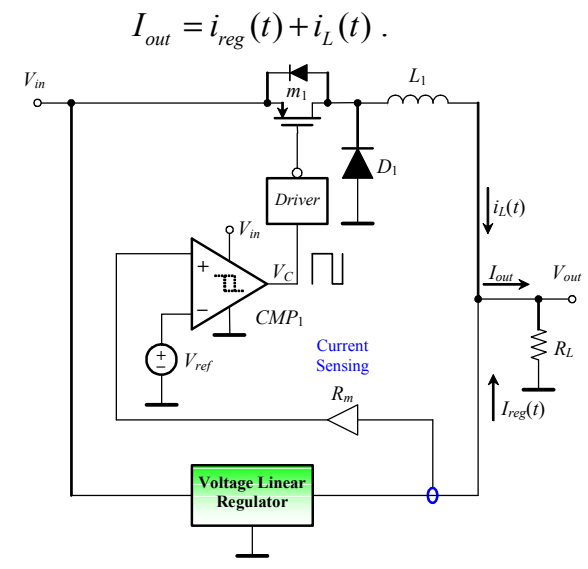

Figure 1. Basic structure of the proposed linear-assisted voltage regulator or hybrid DC-DC regulator.

In a first approximation, consider $C M P_{1}$ without hysteresis. If the load current is below a boundary current, named switching threshold current, $I_{\gamma}$, the output of $C M P_{1}$ is held low. Thus, the switching converter will be disabled and the current through inductor $L_{1}$ will be zero. As a result of this, the linear regulator supplies the load $R_{L}$, providing all the output current $\left(I_{\text {reg }}=I_{\text {out }}\right)$ (Fig. 2). However, when the load current increases and goes slightly beyond this limit current $I_{\gamma}$, comparator output will pass to high level, increasing $i_{L}(t)$ in a linear form. Taking into account (1) and that output current $I_{\text {out }}$ is constant (equal to the quotient $\left.V_{\text {out }} / R_{L}\right), i_{\text {reg }}(t)$ will tend to decrease also linearly, reaching a value below $I_{\gamma}$. At this moment, the comparator changes its output from high to low, cutting off transistor $Q_{1}$ and forcing $i_{L}(t)$ to decrease. Therefore, when $i_{L}(t)$ decreases so that $i_{r e g}(t)>I_{\gamma}, C M P_{1}$ changes from low to high, repeating the cycle again.

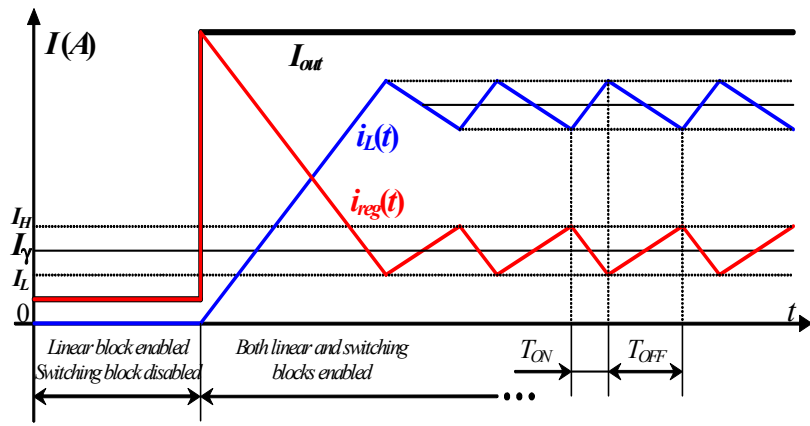

Figure 2. Principle of operation of the proposed linear-assisted DC/DC regulator: Currents through the load (black color), inductance $L_{l}$ (blue) and linear regulator (red).

It is important to highlight that both the reference voltage $V_{\text {ref }}$ and the sensing current element $R_{m}$ determine the switching threshold current $I_{\gamma}$. In this way, the switching instant of the DC/DC converter is controlled by $I_{\gamma}$. This control signal can be adjusted thanks to the gain of the current sensing element, $R_{m}$, and the reference voltage $V_{r e f}$, according to the expression:

$$
I_{\gamma}=\frac{V_{r e f}}{R_{m}} .
$$

With the objective of reducing the dissipated power by the internal transistor of the linear regulator to the utmost, and increasing the efficiency of the whole system, even with significant load currents, this current $I_{\gamma}$ has to be, ideally, equal to zero. However, in a practical voltage regulator, this current should be slightly positive in order not to penalize its good regulation features.

In addition, with the objective of fixing the maximum value of this switching frequency to a suitable value (in order to avoid increasing the switching losses significantly), it is convenient to add a hysteresis to the analog comparator $C M P_{1}$. In this way, designating $V_{H}$ and $V_{L}$ to the upper and lower switching threshold levels of $C M P_{1}$, respectively, the value of the switching frequency $f_{S}$ in steady state is given by:

$$
f_{S}=\frac{R_{m}}{L_{1}} \frac{V_{\text {out }}}{V_{H}-V_{L}}\left(1-\frac{V_{\text {out }}}{V_{\text {in }}}\right) .
$$

On the other hand, the ON time, $T_{O N}$, and OFF time, $T_{O F F}$, in every steady-state switching period is given, respectively, by the following expressions:

$$
T_{\text {ON }}=\frac{L_{1}}{R_{m}} \frac{V_{H}-V_{L}}{V_{\text {in }}-V_{\text {out }}} \text {, and, } T_{\text {OFF }}=\frac{L_{1}}{R_{m}} \frac{V_{H}-V_{L}}{V_{\text {out }}} \text {. }
$$

As an additional advantage, it is important to highlight that typical low-pass filtering capacitors at the output terminal of the switching converters (which capacities can be important in some high-current applications), are not necessary in the proposed structure because the linear regulator implements an efficient low-pass filtering function [12]-[14]. 


\section{DESIGN OF THE PROPOSED LINEAR-ASSISTED REGULATOR}

\section{A. Design of the Linear Voltage Regulator}

The CMOS implementation of the linear voltage regulator used in the topology of the linear-assisted DC-DC regulator is shown in Fig. 3. It consists of a classical three-stage operational amplifier. On the one hand, the first stage is a $p$ MOS differential input pair ( $m_{19}$ and $m_{20}$ transistors) with an $n$-channel current mirror active load ( $m_{21}$ and $m_{22}$ transistors). Transistor $m_{23}$ provides the suitable bias current for this differential pair. On the other, the second gain stage is a simple CMOS inverter with $m_{24}$ as the driver of the output stage, and $m_{25}$ being its active load. The output of this second stage is connected to its input by means of a Miller compensation capacitance $C_{C}$ in order to assure regulator stability. Finally, the output stage is a classical class-B pushpull output buffer (transistors $m_{26}$ and $m_{27}$ ). It should be noted that this final stage (in particular, the ratio $W / L$ of the output transistors) determines the output current capability of the linear regulator. In the presented case, for the considered maximum output current, this ratio is $300 \mu \mathrm{m} / 0.36 \mu \mathrm{m}$. These two devices are transistors with low threshold voltage $\left(V_{t}\right)$, in order to improve their input dynamic ranges (gate-source voltage).

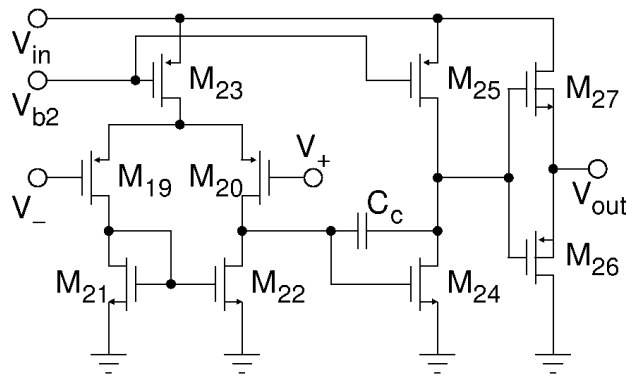

Figure 3. Circuit of the CMOS operational amplifier used in the linearassisted regulator.

\section{B. Design of the Analog Comparator}

The main core of the controller is an analog comparator with a suitable hysteresis. This analog comparator is presented in Fig. 4. It consists of a first stage, an $n$-channel differential input pair (transistors $m_{1}$ and $m_{2}$ ), with their associated current mirrors that act as active loads $\left(m_{4}\right.$ and $\left.m_{5}\right)$, and transistor $m_{3}$, a current source that provides the bias current for the differential pair. The output of this first stage is applied to a second one $\left(m_{6}\right.$ to $\left.m_{11}\right)$ that assures a suitable hysteresis to the analog comparator thanks to transistors $m_{6}$ to $m_{9}$. Its output is applied to a second differential pair $\left(m_{12}\right.$ to $\left.m_{16}\right)$ that boosts the total gain of the comparator. Finally, the fourth stage, transistors $m_{17}$ and $m_{18}$, assures the output current capability. They have a reasonable value for the transistor widths in order to drive the switch gates of the switching DC-DC converter.

\section{Complete Linear-Assisted Regulator}

Finally, Fig. 5 shows the final implementation of the CMOS linear-assisted regulator, in which the operational amplifier $O A$ is given in Fig. 3, and the analog comparator $C M P$ is shown in Fig. 4. Resistors $R_{1}$ and $R_{2}$ determine the reference voltage $V_{\text {ref }}$ given in Fig. 1. Notice that this voltage fixes the value of the switching threshold current $I_{\gamma}$ (according to (2)), limiting the maximum current through the linear regulator at the steady state. Finally, transistors $M_{p}$, and $M_{n}$, diodes $D_{1}$ and $D_{2}$, and an off-chip inductor $L$ define the buck converter structure. Although transistor $M_{n}$ could be removed from the circuit, the linear-assisted DC-DC regulator includes a synchronous buck converter with transistors $M_{p}$ and $M_{n}$. The use of the synchronous step-down converter improves the total efficiency of the linear-assisted regulator.

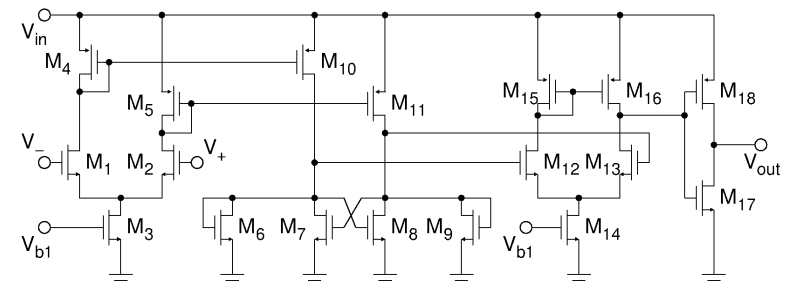

Figure 4. Proposal of the circuit for the CMOS analog comparator used in the control loop of the switching DC-DC converter.

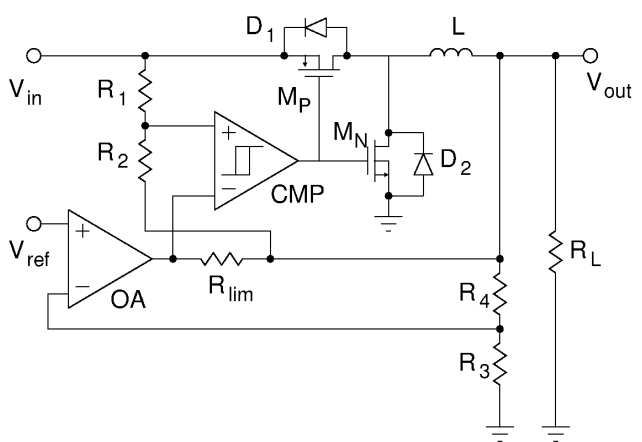

Figure 5. CMOS implementation of the linear-assisted DC-DC regulator.

\section{Simulation Results}

With the objective of validating the correct operation of the proposed circuital structure, the CMOS on-chip linearassisted regulator presented in Fig. 5 has been simulated using a standard CMOS $0.18-\mu m$ technology. The parameters of this model have been obtained from UMC (United Microelectronics Corporation $^{B}$ ). The design must guarantee the power supply for an analog design included in the same integrated circuit with a constant voltage of $1.1 \mathrm{~V}$. In our case, this load is an analog design that consists of a low-power continuous time filter for MEMS signal filtering with its central-frequency and quality-factor control loops. The regulator input voltage (chip input voltage) can vary from 1.6 $V$ to $1.8 \mathrm{~V}$. The total consumption of the aforementioned load is around $15 \mathrm{~mA}$.

In order to corroborate and validate the functionality of the proposed linear-assisted structure, Fig. 6 shows the voltage and current waveforms of the topology at the steady state. In particular, the output voltage $V_{\text {out }}$ (top trace), the comparator output $V_{C M P}\left(\right.$ middle), and load $i_{\text {out }}(t)$, inductor $i_{L}(t)$ and linear regulator $i_{\text {reg }}(t)$ currents (bottom axis).

Finally, Fig. 7 shows the transient response of the same voltage and current waveforms shown in the previous figure, when the load current is a sinusoidal pulse between 0 and 15 
$m A$. Notice that when the output current is below the threshold current $\left(I_{\text {out }}<I_{\gamma}\right)$, the output current is provided by the linear regulator. However, when $I_{\text {out }}>I_{\gamma}$, the inductor current follows the load current; that is, almost all the load current is provided by the switching converter. In addition, the output voltage is determined by the linear regulator, which guarantees a good voltage regulation and only provides a small percentage of the total load current, remaining a good efficiency of the whole linear-assisted regulator.

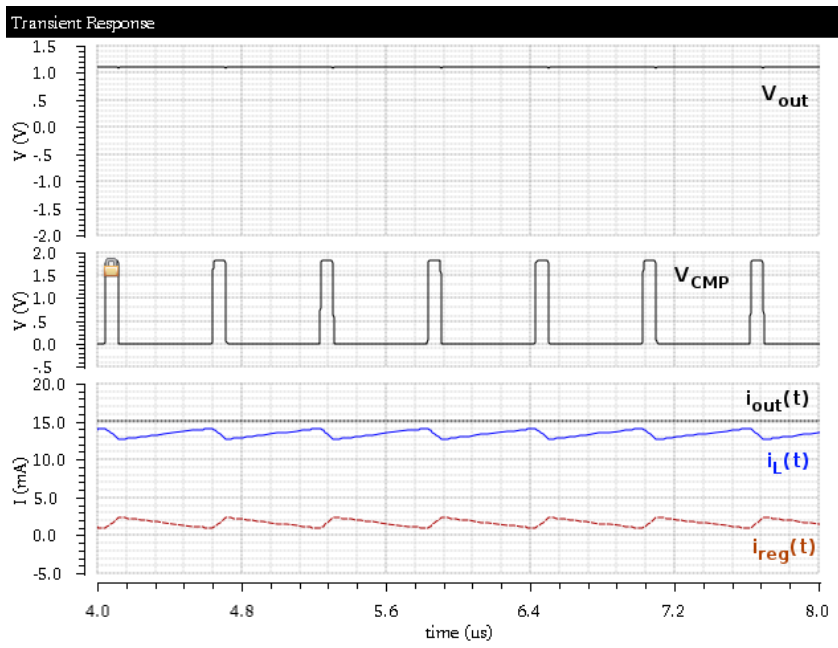

Figure 6. Voltage and current waveforms of the proposed linear-assisted DC-DC regulator at the steady state.

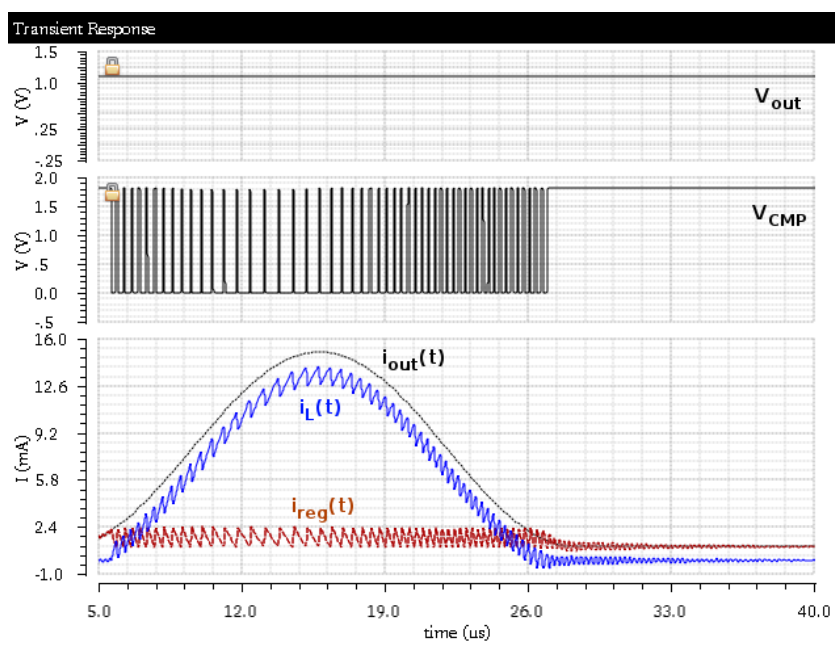

Figure 7. Voltage and current waveforms of the proposed linear-assisted DC-DC regulator when load current is a sinusoidal pulse.

\section{CONCLUSIONS}

Taking into account the advantages of the CMOS technology to implement on-chip power supply subsystems for integrated circuits, this article has shown the design of an on-chip CMOS implementation of a hybrid or linear-assisted DC-DC regulator. On the one hand, the article has shown the suitable regulation features of the output voltage for the presented structure with low-to-medium current consumption. On the other hand, the efficiency of the whole structure should be hardly penalized for the limitations of the linear regulators.
Besides, the inclusion of a linear regulator in parallel with the switching converter achieves a good performance with fast responses to transients of load current and input voltage. Note that without output capacitance, the linear-assisted regulator achieves good dynamic and static characteristics.

Simulation results have demonstrated the feasibility to make an excellent and simple on-chip CMOS linear-assisted DC-DC regulator. Surely, the final experimental implementation of the on-chip regulator will degrade some specification. However, this technology allows being optimist for its expansion into commercial integrated circuits.

\section{ACKNOWLEDGMENT}

This work has been partially supported by the Spanish Ministry of Science and Innovation by projects TEC201015765/MIC, and TEC2011-27047.

\section{REFERENCES}

[1] R. K. Dokania, and G. A. Rincón-Mora, "Cancellation of Load Regulation in Low Drop-Out Regulators", Electronic Letters, vol. 38 $\left(n^{\circ} 22\right)$, pp. 1300-1302, $24^{\text {th }}$ October 2002.

[2] V. Grupta, G. A. Rincón-Mora, and P. Raha, "Analysis and Design of Monolithic, High PSR, Linear Regulator for SoC Applications", Proceedings of the IEEE International SoC Conference, pp. 311-315, 2004.

[3] R.J. Milliken, J. Silva-Martinez, and E. Sanchez-Sinencio, "Full OnChip CMOS Low-Dropout Voltage Regulator", IEEE Transactions on Circuits and Systems I: Regular Papers, vol. 54 (n ${ }^{\circ}$ 9), pp. 1879-1890, September 2007.

[4] C.K. Chava, and J. Silva-Martinez, "A Frequency Compensation Scheme for LDO Voltage Regulators", IEEE Transactions on Circuits and Systems I: Regular Papers, vol. 51 ( $\mathrm{n}^{\circ}$ 6), pp. 1041-1050, June 2004.

[5] R. W. Erickson, and D. Maksimovic, "Fundamentals of Power Electronics", 2nd edition, Ed. Kluwer Academic Publishers, 2001.

[6] J. G. Kassakian, M. F. Schlecht, and G. C. Verghese. "Principles of Power Electronics”. Ed. Addison-Wesley, 1991.

[7] V. Yousefzadeh, E. Alarcon, and D. Maksimovic, "Band Separation and Efficiency Optimization in Linear-Assisted Switching Power Amplifiers", $37^{\text {th }}$ IEEE Power Electronics Specialists Conference (PESC'06), pp. 1-7, 18-22 Jun. 2006.

[8] B. Arbetter, and D. Maksimovic, "DC-DC Converter with Fast Transient Response and High Efficiency for Low-Voltage Microprocessor Loads", $13^{\text {th }}$ Annual IEEE Applied Power Electronics Conference and Exposition (APEC'98), vol. 1, pp. 156-162. 15-19 Feb. 1998.

[9] P. Midya and F. H. Schlereth, "Dual Switched Mode Power Converter", IECON. Industrial Electronics Society, pp. 155-158, 1989.

[10] F. H. Schlereth and P. Midya, "Modified Switched Power Convertor with Zero Ripple", Proceedings of the 32nd IEEE Midwest Symposium on Circuits and Systems (MWSCAS'90), pp. 517-520, August 1990.

[11] H. Ertl, J. W. Kolar and F. C. Zach, "Basic Considerations and Topologies of Switched-Mode Assisted Linear Power Amplifiers", IEEE Transactions on Industrial Electronics, vol. $44\left(n^{\circ} 1\right)$ : pp. 116123, February 1997.

[12] A. Conesa, H. Martínez, and J.M. Huerta, "Dynamic Analysis of Hybrid DC-DC Converters", $12^{\text {th }}$ European Conference on Power Electronics and Applications (EPE 2007), September 2007.

[13] H. Martínez, and A. Conesa, "Modeling of Linear-Assisted DC-DC Converters", 18th European Conference on Circuit Theory and Design, (ECCTD 2007), Desember 2007.

[14] H. Martínez, and A. Conesa, "Linear-Assisted DC-DC Converters Based on CMOS Technology", IEEE Power Electronics Specialists Conference (PESC 2008), June 2008. 